\title{
Ultra-Selective Polyamide Membrane from Metal Organic Framework Assembly Regulated Interfacial Polymerization for Desalination and Water Reuse
}

Yue Wen

Tongji University

Ruobin Dai

Tongji University

Xuesong Li

Tongji University

Xingran Zhang

Tongji University

Xingzhong Cao

Institute of High Energy Physics https://orcid.org/0000-0001-5011-5912

Zhichao Wu

Tongji University

Shihong Lin

Vanderbilt University https://orcid.org/0000-0001-9832-9127

Chuyang Tang

University of Hong Kong

Zhiwei Wang ( $\nabla$ zwwang@tongji.edu.cn )

Tongji University https://orcid.org/0000-0001-6729-2237

\section{Article}

Keywords: polyamide membrane, metal-organic framework nanoflakes, desalination

Posted Date: August 10th, 2021

DOl: https://doi.org/10.21203/rs.3.rs-798562/v1

License: (c) (i) This work is licensed under a Creative Commons Attribution 4.0 International License.

Read Full License 


\section{Ultra-Selective Polyamide Membrane from Metal Organic}

\section{Framework Assembly Regulated Interfacial Polymerization for}

\section{Desalination and Water Reuse}

Yue Wen ${ }^{\dagger}$, Ruobin $\mathrm{Dai}^{\dagger}$, Xuesong $\mathrm{Li}^{\dagger}$, Xingran Zhang ${ }^{\dagger}$, Xingzhong Cao ${ }^{\S}$, Zhichao $\mathrm{Wu}^{\dagger}$,

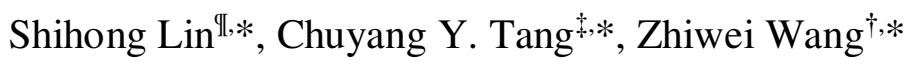

${ }^{\dagger}$ State Key Laboratory of Pollution Control and Resource Reuse, Shanghai Institute of Pollution Control and Ecological Security, School of Environmental Science and Engineering, Tongji University, 1239 Siping Road, Shanghai 200092, China

${ }^{\S}$ Institute of High Energy Physics, CAS, Beijing 100049, China

IDepartment of Civil and Environmental Engineering, Vanderbilt University, Nashville, Tennessee 37235-1831, United States

* Department of Civil Engineering, the University of Hong Kong, Pokfulam Road, Hong Kong S.A.R., China

Submitted to Nature Communications (August, 2021)

*Corresponding Author.

Email: shihong.lin@vanderbilt.edu (S. Lin);

tangc@hku.hk (C. Tang);

zwwang@tongji.edu.cn (Z. Wang). 


\begin{abstract}
While reverse osmosis (RO) is the leading technology to address the challenge of water scarcity through desalination and potable reuse of wastewater, current RO membranes still fall short in rejecting certain harmful constituents from seawater (e.g., boron) and wastewater (e.g., $\mathrm{N}$-nitrosodimethylamine, or NDMA). In this study, we develop an ultraselective polyamide (PA) membrane via enhancing interfacial polymerization using amphiphilic metal-organic framework (MOF) nanoflakes. In this process, MOF nanoflakes horizontally aligned at the water/hexane interface to accelerate the transport of diamine monomers across the interface and conserve gas bubbles and heat of reaction near the interface. These mechanisms synergistically led to the formation of a crumpled PA nanofilm with an ultrathin intrinsic thickness of $\sim 5 \mathrm{~nm}$ and a high cross-linking degree of $97 \%$. The resulting PA membrane delivered excellent desalination performance that is beyond the existing upper-bound of perm-selectivity, and exhibited very high rejection (>90\%) of boron and NDMA unmatched by state-of-the-art RO membranes.
\end{abstract}




\section{INTRODUCTION}

The escalating global water shortage motivates sustainable water resource management for supplying clean, safe, and adequate water ${ }^{1,2}$. To date, reverse osmosis (RO) has become a leading technology to address the severe shortages in fresh water supply through desalination and wastewater reuse $\mathrm{e}^{3,4}$. Although existing polyamide (PA) RO membranes exhibit high water-salt selectivity with salt rejection beyond $99 \%$ (for seawater desalination), they remain inadequate in removing certain harmful and regulated constituents from feedwater ${ }^{5,6}$.

For example, $N$-nitrosodimethylamine (NDMA) is a carcinogenic disinfection byproduct commonly found in the RO permeate of wastewater reuse plants ${ }^{7}$. Historically, high concentrations of NDMA (400,000 ppt on site and 20,000 ppt offsite) had been found in aquifers in southern California where RO-recycled wastewater was used for groundwater replenishment, which prompts California to implement a stringent action level for NDMA as low as 10 ppt. $^{8}$ Unfortunately, commercial RO membranes show unsatisfactory rejection of NDMA (30-80\% for lab-scale tests ${ }^{9-11}$ and merely 5-60\% at pilot- to full-scale levels ${ }^{12}$ ), which necessitates highly energy-intensive advanced oxidation processes (e.g., ultraviolet coupled with peroxide) to destroy NDMA in RO permeates ${ }^{13,14}$. The poor rejection of NDMA is attributed to its small molecular weight $\left(74 \mathrm{~g} \mathrm{~mol}^{-1}\right)$, uncharged state at $\mathrm{pH}$ 6-8, and strong dipole moment $(6.3 \text { Debye })^{13,15}$. Another challenge faced by commercial RO membranes in seawater desalination is the inadequate removal of boron that is neutral (as $\mathrm{B}(\mathrm{OH})_{3}$, molecular weight: $\left.61.8 \mathrm{~g} \mathrm{~mol}^{-1}\right)$ in seawater. Due to the low boron rejection (approximately $40-80 \%$ ) by commercial RO membranes ${ }^{16,17}$, the desalinated permeate often requires an additional second-pass RO treatment, which increases the cost, energy 
consumption, and footprint of seawater desalination plants. The poor rejection of these important species by commercial RO membranes calls for the development of ultraselective RO membranes for more cost-effective seawater desalination and wastewater reuse.

Recent studies have shown that steric hindrance plays a critical role in determining transport through RO membranes ${ }^{10,11}$. Efforts have been dedicated to decrease the free volume of PA layers, involving heat treatment ${ }^{5,18}$ and surface modification (e.g., surface coating or 'plugging' $)^{9,19}$. However, the improvement of solute rejection via these approaches is at the expense of water permeability $y^{5,9,20}$. While other efforts to fabricate more selective membranes have been attempted recently, such as incorporating porous nanomaterials $^{21,22}$, stacking two-dimensional (2D) nanoflakes ${ }^{23,24}$, and integrating biomimetic nanochannels ${ }^{1,25,26}$, no study has thus far reported the production of defectfree membrane that effectively rejects small neutral solutes such as NDMA and boron. Additionally, the scalable fabrication of membranes based on novel materials still face significant challenges.

The performance of the thin-film composite (TFC) RO membrane is primarily dependent on the PA active layer ${ }^{27}$, which is fabricated via an interfacial polymerization (IP) reaction between an amine monomer, e.g., m-phenylenediamine (MPD), and an acyl chloride monomer, e.g., trimesoyl chloride (TMC) ${ }^{28}$. As the IP process involves extremely fast and uncontrolled reaction at the water/oil interface ${ }^{22,29}$, the formation of the PA active layer with both fast water transport and outstanding solute rejection remains as a substantial technical challenge. 
Amphiphilic nanoflakes, which possess highly asymmetric surface wettability and distinct physicochemical properties ${ }^{30,31}$, have garnered considerable attention in drug delivery ${ }^{32}$, catalysis ${ }^{33}$, and oil recovery ${ }^{34}$. Inspired by their great potential in interfacial properties regulation $^{35}$, herein, we develop an ultra-selective polyamide $\mathrm{RO}$ membrane by enhancing the IP process at the interface to create a crumpled, free-standing, and ultrathin nanofilm with an intrinsic thickness of $\sim 5 \mathrm{~nm}$ and a high cross-linking degree of $\sim 97 \%$. The amphiphilic metal-organic framework (MOF) nanoflakes self-assemble at the water/hexane interface and regulate the heat dissipation and the transport of MPD monomers during IP (Figure 1). This process, namely MOF assembly regulated IP (MARIP), results in an ultrathin PA nanofilm with unprecedentedly high rejections of NDMA (90.3 $\pm 0.4 \%)$ and boron (90.1 $\pm 3.4 \%)$. The mechanisms of amphiphilic nanoflakes in enhancing IP were further investigated using doppler broadening energy spectroscopy (DBES), isothermal titration calorimetry and molecular simulations. 
(a)

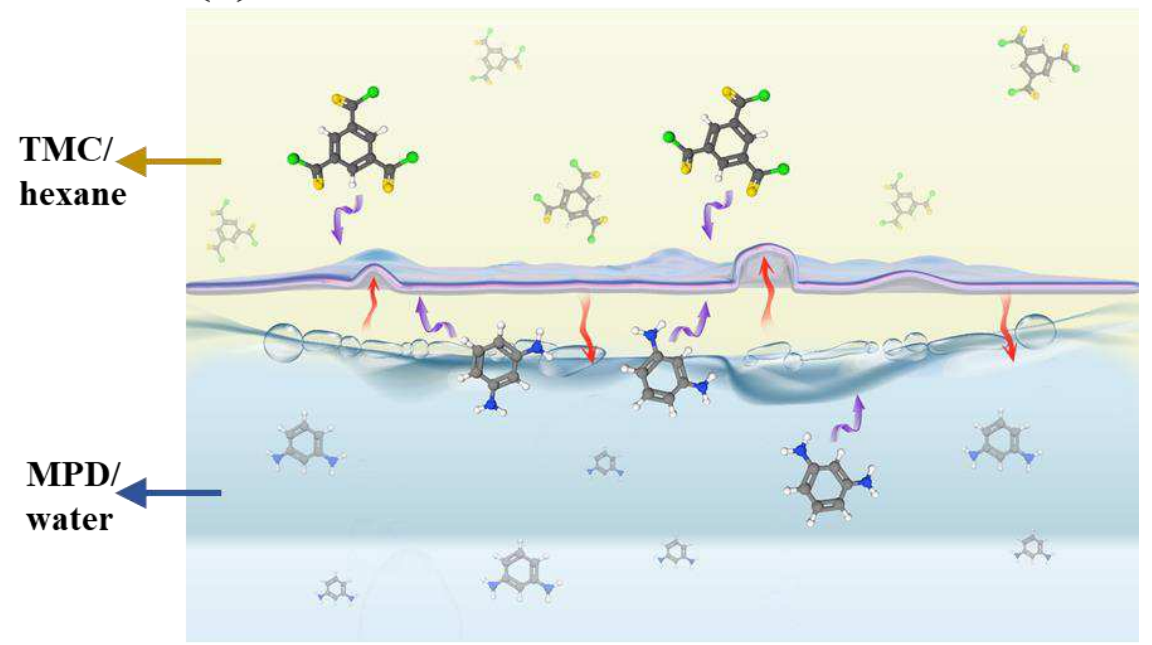

(b)

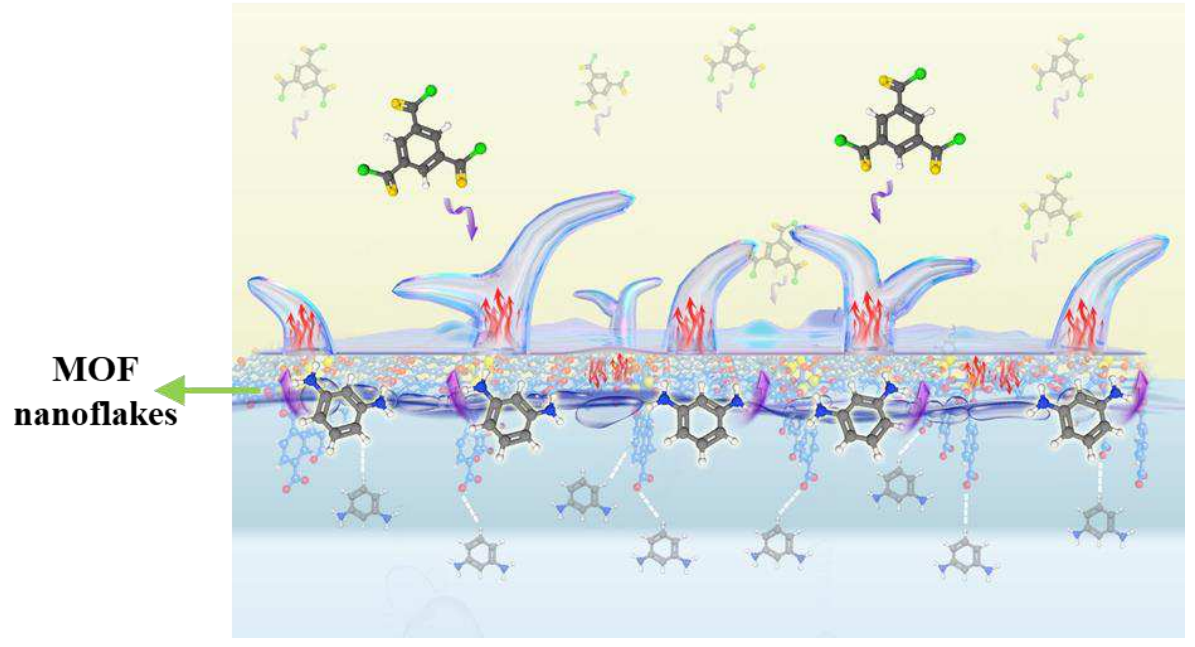

Figure 1. Graphical illustration of (a) IP process at a free interface (FIP) and (b) IP process at a MOF-regulated interface, i.e., MOF assembly regulated IP (MARIP), where the amphiphilic MOF nanoflakes conserved heat locally and accelerated MPD transport across the water/hexane interface to form a crumbled PA active layer with a smaller intrinsic thickness and a higher degree of cross-linking. 


\section{RESULTS}

Formation of free-standing polyamide nanofilm via MARIP. CuBDC MOF were used as the amphiphilic nanoflakes for regulating the IP process due to their large porosity, high aspect ratio, and asymmetric wettability (Supplementary Figure 2). The large difference in surface energy between the hydrophilic segments (i.e., - $\mathrm{COOH}$ groups from BDC on the edges of nanoflakes) and the hydrophobic segments (i.e., aromatic and aliphatic sites from BDC organic linkers exposed in the lateral dimensions) led to strong adsorption of CuBDC at the interface, resulting in an interfacial self-assembly of the MOF nanoflakes. ${ }^{36}$ The formation of interfacial MOF layer was corroborated by the exceptional ability of MOF nanoflakes to stabilize oil/water mixture: when MOF nanoflakes were added in an oil/water mixture, stable oil-in-water emulsions formed spontaneously and almost immediately (Supplementary Figure 3a) as the MOF nanoflakes effectively reduced the interfacial tension (Supplementary Figure 3b) ${ }^{37}$. The self-assembly of MOF nanoflakes at the interface was also supported by molecular dynamics simulation that showed the alignment of nanoflakes along the interface (see details in Supplementary Materials and Supplementary Movie 1).

The ultrathin PA nanofilm was fabricated using MARIP at a support-free water/hexane interface with the interfacial self-assembly of MOF nanoflakes (Supplementary Figure 4 and Supplementary Figure 5). The fabricated PA nanofilm was then transferred onto a polyethersulfone substrate for further tests. The PA membranes obtained with MOF dosages of 0.05 wt. $\%, 0.10 \mathrm{wt} . \%$, and $0.20 \mathrm{wt} . \%$ are denoted as MARIP-0.05, MARIP-0.1, and MARIP-0.2, and the membrane formed via free interface polymerization without MOF was used as a control (named as FIP). To identify the position of MOF nanoflakes in the 
water/hexane system relative to that of the PA layer, the formed PA nanofilms was further analyzed by XPS and XRD. Cu element was not detected on the top surface of all the PA nanofilms (Supplementary Figure 6a) but became increasingly abundant at the bottom surface with increasing MOF dosage (Supplementary Figure 6b). XRD patterns (Supplementary Figure 6c) on the bottom surface of PA nanofilms formed via MARIP exhibited the $(\overline{4} 02)$ crystallographic plane of the MOF structure, suggesting that MOF nanoflakes align in parallel to the bottom surface of the nanofilm.

Morphology and properties of polyamide nanofilms from MARIP. The PA film fabricated using MARIP are morphologically very different from the PA film fabricated using FIP without MOF mediation (Figure 2a, $2 \mathrm{~b}$ and Supplementary Figure 7). Compared with the smooth PA film formed at the free interface without MOF, PA films formed via MARIP have "ridge-and-valley" structures with substantially increased surface roughness (Supplementary Figure 8a). The distinct morphological differences are associated with the different IP processes: in FIP, the heat released from the reaction of MPD and TMC rapidly dissipated across the free interface; in MARIP, however, the MOF nanoflakes acted as a barrier for heat dissipation across the interface and induced a significant local temperature gradient that caused interfacial hydrodynamic instability of the hexane layer driven by Rayleigh-Bénard convection ${ }^{38,39}$. The accumulation of heat where polymerization occurred led to the local formation of gas nanobubbles that roughened the PA nanofilms ${ }^{28,39}$. 
(a)
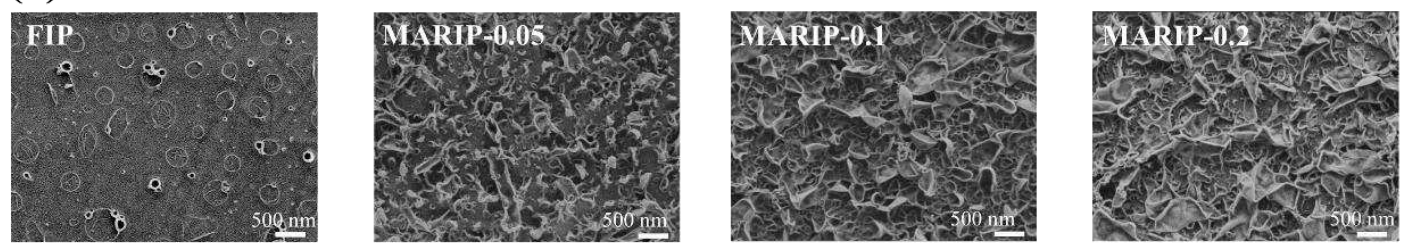

(b)
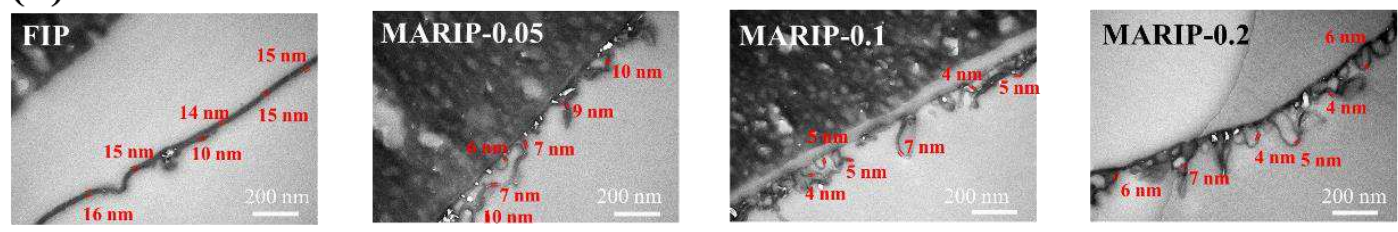

(c)

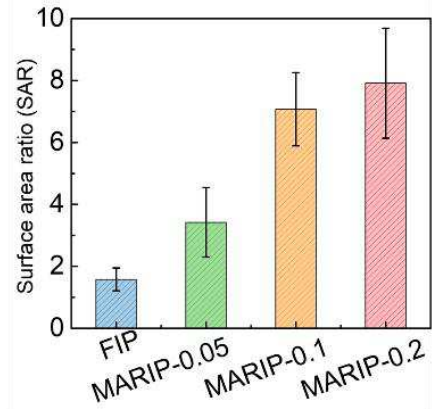

(d)

(e)
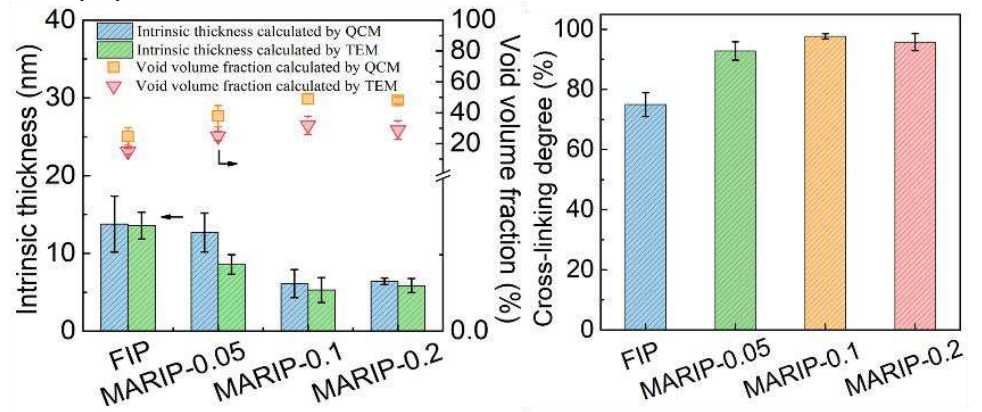

(f)

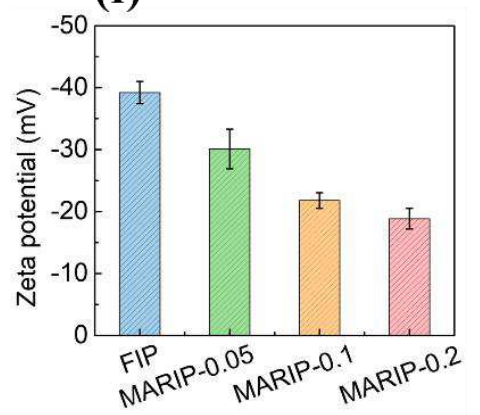

(g)

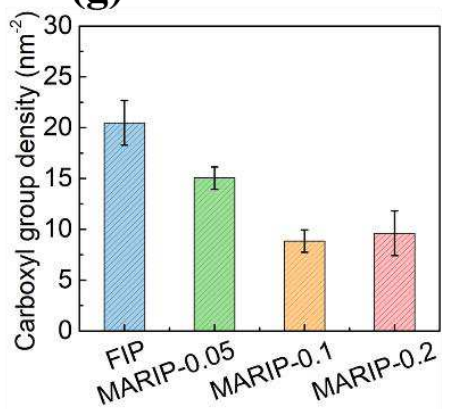

(h) Mean depth (nm)

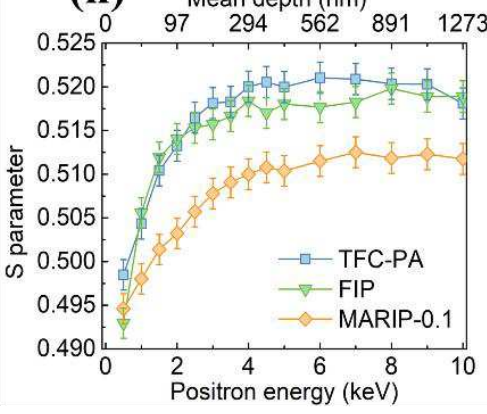

Figure 2. Morphology and properties of FIP, MARIP-0.05, MARIP-0.1, and MARIP-0.2 membranes. (a) SEM micrographs of the top surfaces; (b) TEM micrographs of the cross sections; (c) the surface area ratio (SAR) from analysis of TEM images $(n=10)$; (d) the intrinsic thickness and void volume fraction measured from QCM $(n=3)$ and TEM $(n=10)$; (e) degree of cross-linking; (f) zeta potential ( $n=3)$; (g) carboxyl group density $(n=6)$; and (h) evolution of $S$ parameters for the PA nanofilms obtained from conventional IP with support layer (TFC-PA), FIP, and MARIP (0.1\% MOF). 
To confirm the role of MOF nanoflake assembly in MARIP, we further fabricated MixMOF PA membrane by adding TMC/hexane solution to the system immediately (within 5 s) after MOF nanoflakes were added to water (without forming an interfacial selfassembly). Without horizontal alignment at the interface, the MOF nanoflakes were incorporated into the nanofilm during the IP process. In the absence of interfacial confinement by the self-assembled MOF layer, the Mix-MOF PA membrane resembled those of the FIP membrane (Supplementary Figure 9a and Supplementary Figure 9b) and did not exhibit the ridge and valley structure observed in PA membranes formed via MARIP. Both the permeance and salt rejection of Mix-MOF membrane were inferior to PA membranes fabricated using MARIP (Supplementary Figure 9c).

The surface area ratio (SAR) of the nanofilm, i.e., the ratio of the three-dimensional surface area over the projected area, was estimated from the TEM images. SAR increased dramatically from $1.6 \pm 0.4$ for FIP membrane to $3.4 \pm 1.1,7.1 \pm 1.2$ and $7.9 \pm 1.8$ for MARIP0.05, MARIP-0.1, and MARIP-0.2 membranes, respectively (Figure 2c). The increased SAR is likely caused by the presence of more nanovoids (Figure $2 \mathrm{~b}$ and Figure $2 \mathrm{~d}$ ). These nanovoids enhance water uptake ${ }^{40,41}$ and provide a large effective area for water permeation, thus resulting in a higher water permeability for PA membranes derived from MARIP. The SEM micrographs of the bottom surface show that both the quantity and size of the openings increased with a larger dosage of MOF nanoflakes (Supplementary Figure 10 and Supplementary Figure 8b), which could provide more channels for water transport through the PA nanofilm. In addition to a larger SAR, the TEM cross-sectional images (Figure 2b and Supplementary Figure 7) also show that the intrinsic thickness decreased significantly from $13.6 \pm 1.7 \mathrm{~nm}$ for the FIP film to $8.6 \pm 1.3 \mathrm{~nm}, 5.3 \pm 1.6 \mathrm{~nm}$, and $5.9 \pm 0.9$ 
nm for MARIP-0.05, MARIP-0.1, and MARIP-0.2 nanofilms from MARIP, respectively (Figure 2d). The intrinsic thickness of the PA films represents the characteristic length for trans-membrane water transport ${ }^{39,42}$ and differs from the apparent thickness (i.e., the thickness of the crumpled polyamide structure) which increased with MOF nanoflakes addition (Supplementary Figure 11). The PA films fabricated using MARIP were much thinner than the PA active layers in thin-film composite (TFC) membranes fabricated using conventional IP (25 35 nm, Supplementary Figure 12) or PA nanofilms reported elsewhere ${ }^{42,43}$.

Furthermore, the enhanced transport of MPD due to reduced free energy barrier ${ }^{44}$ substantially improved the cross-linking degree of PA nanofilms (Figure 2e based on XPS results) from $74.9 \pm 3.9 \%$ for FIP film to $92.8 \pm 3.1 \%, 97.7 \pm 0.9 \%$ and $95.7 \pm 2.9 \%$ for MARIP-0.05, MARIP-0.1, and MARIP-0.2 nanofilms, respectively. The increased degree of crosslinking is also consistent with the less negative zeta potential (Figure $2 \mathrm{f}$ ) and the reduced carboxyl group density (Figure $2 \mathrm{~g}$ ). The extremely high cross-linking degree led to a significantly higher elastic modulus compared to that of PA nanofilms reported elsewhere $^{42}$ (Supplementary Figure 13 and Supplementary Table 1), and more importantly, better solute rejection ${ }^{45}$. The denser PA nanofilm from MARIP was further confirmed by Doppler broadening energy spectroscopy (DBES). As a lower $S$ parameter represents a smaller free volume (or sub-nanometer pores), ${ }^{46}$ the consistently lower range of the $S$ parameter of MARIP-0.1 as compared to that of PA films formed in FIP or conventional IP with support layer indicates that MARIP-0.1 has a smaller free volume (Figure $2 \mathrm{~h}$ ). 
Separation performance of polyamide nanofilms from MARIP. The separation performance of the PA membranes was evaluated using crossflow filtration. The FIP membrane had a relatively low water permeance and low salt rejection, even lower than that of TFC-PA membrane prepared via conventional IP with a support layer. In contrast, the water permeance of the MARIP-0.1 and MARIP-0.2 membranes was $262 \%$ and $277 \%$ of that of the FIP membrane, respectively (Figure 3a). The dramatic permeance enhancement for PA membranes formed from MARIP was attributed to: (i) the larger effective filtration area for water transport and (ii) the lower intrinsic thickness of the active layer that reduces the water transport distance. As shown in Figure 3a, the MARIP membranes also exhibited excellent salt rejection performance. Notably, the $\mathrm{NaCl}$ rejection was dramatically improved from $95.4 \pm 0.5 \%$ (FIP) to $99.5 \pm 0.1 \%$ (MARIP-0.1) and 99.1 $\pm 0.2 \%$ (MARIP-0.2), as a result of the higher cross-linking degree of the PA membranes from MARIP. Compared with the upper bound of the trade-off between water permeance and water/ $\mathrm{NaCl}$ selectivity for existing lab-made and commercial $\mathrm{RO}$ membranes (Figure 3b, see details in Supplementary Materials Section 2.4), the MARIP0.1 and MARIP- 0.2 membranes are both substantially more permeable and selective at the same time, surpassing the upper bound of perm-selectivity tradeoff. 
(a)

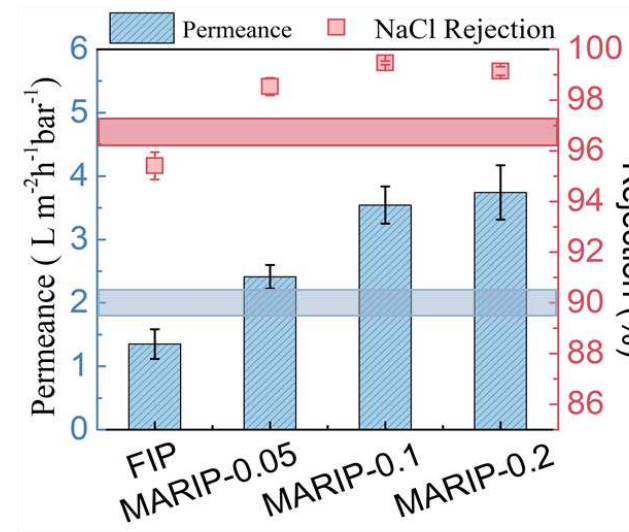

(c)

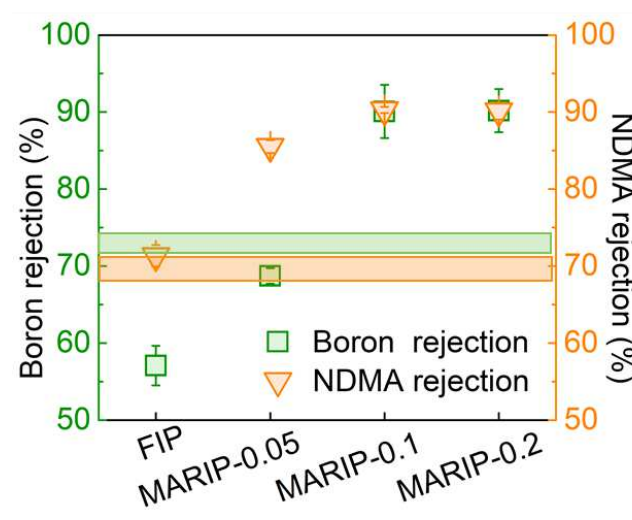

(b)

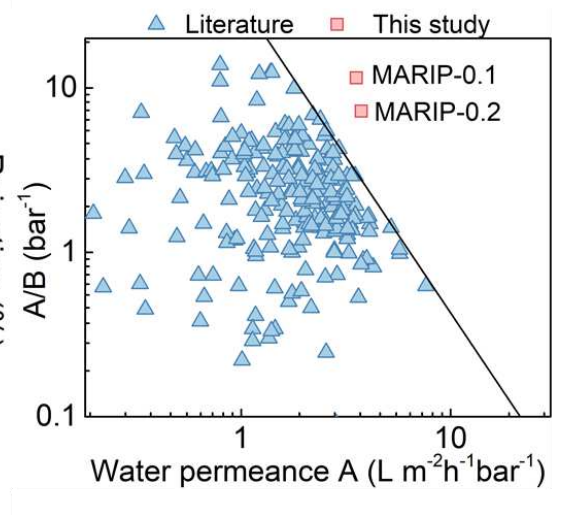

(d)

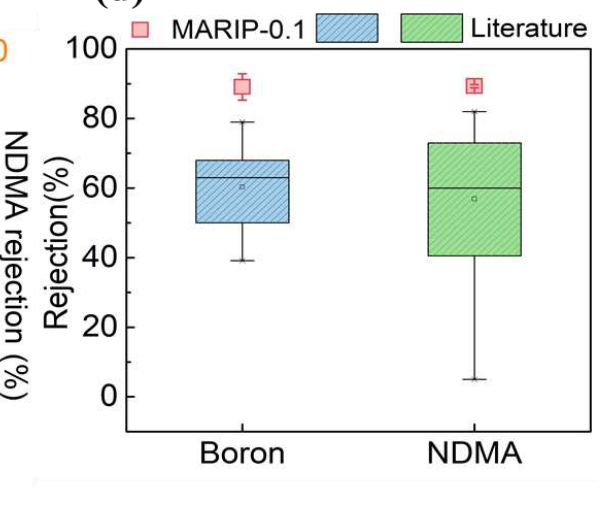

Figure 3. Membrane separation performance. (a) Pure water permeance and $\mathrm{NaCl}$ rejection $\left(2000 \mathrm{mg} / \mathrm{L} \mathrm{NaCl}, 16 \mathrm{bar}, 24^{\circ} \mathrm{C}\right)(n=6)$. The blue and red horizontal bars represent the water permeance and $\mathrm{NaCl}$ rejection of conventional TFC-PA membrane, respectively (the thickness represents standard deviation). (b) Comparison of the separation performance of MARIP-0.1 and MARIP-0.2 membranes with the upper bound of the trade-off between pure water permeance and water/ $\mathrm{NaCl}$ selectivity reported. (c) Rejection of boron $(5 \mathrm{mg} \mathrm{L}$ ${ }^{1}$, pH 6.0, 16 bar, $\left.24^{\circ} \mathrm{C}\right)$ and NDMA $\left(890 \mu \mathrm{g} \mathrm{L}^{-1}, 16 \mathrm{bar}, 24^{\circ} \mathrm{C}\right)(n=6)$. The green and orange horizontal bars represent the rejection boron and NDMA by conventional TFC-PA membranes, respectively. (d) Comparison of the boron and NDMA rejection performance of MARIP-0.1 with data reported in literature. 
The water/solute selectivity, especially for very small neutral molecules, is of great significance for RO applications in desalination and potable reuse of wastewater ${ }^{13}$. The FIP membrane exhibits low rejection of boron $(57.1 \pm 2.6 \%)$ and NDMA $(71.3 \pm 1.4 \%)$ due to its low degree of cross-linking (Figure 3c). In contrast, much higher rejection of both species was achieved with the MARIP membranes. Specifically, MARIP-0.1 and MARIP-0.2 membranes exhibited extremely high rejection of NDMA $(90.3 \pm 0.4 \%$ and $90.1 \pm 1.1 \%$, respectively) and boron (90.1 $\pm 3.4 \%$ and $90.2 \pm 2.8 \%$, respectively) as a result of the high degree of cross-linking. Compared with the lab-scale, pilot-scale, and full-scale TFC RO membranes in recent literature (Figure 3d), the MARIP-0.1 membrane exhibited unprecedentedly high rejection of both boron and NDMA. In particular, the boron rejection by MARIP-0.1 membrane tested at $\mathrm{pH} 6$ was higher than most of the RO membranes tested at $\mathrm{pH} 7$, even though boron rejection generally increases with increasing $\mathrm{pH}^{47}$. With the MARIP-0.1 membrane, a single-pass seawater RO can produce water with a sufficiently low boron level that meets the irrigation and drinking water standard of World Health Organization $(\mathrm{WHO})^{48}$, which can translate to a substantial reduction of capital cost by eliminating the second-pass RO dedicated for boron removal in conventional seawater RO plants.

\section{DISCUSSION}

Interfacial polymerization (IP) is a complex and non-equilibrium process, where two monomers polymerize rapidly and irreversibly near the interface between two immiscible phases $^{49}$. In MARIP, the amphiphilic MOF nanoflakes were used to enhance IP process to obtain an ultra-selective PA layer with simultaneous improvement of water permeance and 
selectivity. We hypothesize that the ultra-selectivity of PA membranes from MARIP is attributable to the combined effects of (i) accelerated IP reaction resulting from increased interfacial temperature due to hindered heat dissipation, and (ii) facilitated trans-interface transport of MPD caused by the reduced energy barrier (Figure 4) and enhanced electrostatic attraction at the interface.

(a)

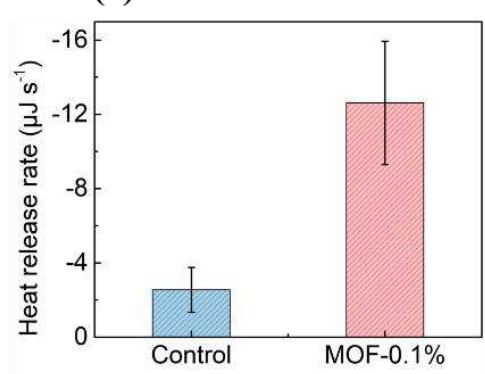

(d)

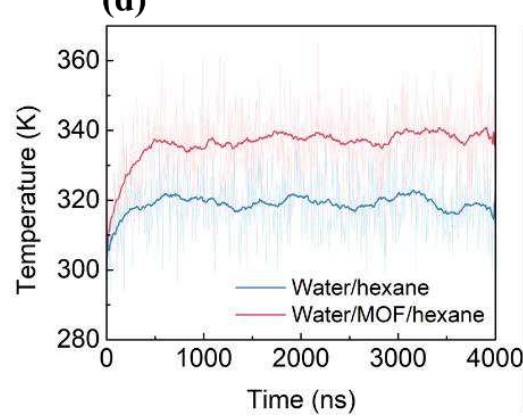

(b)

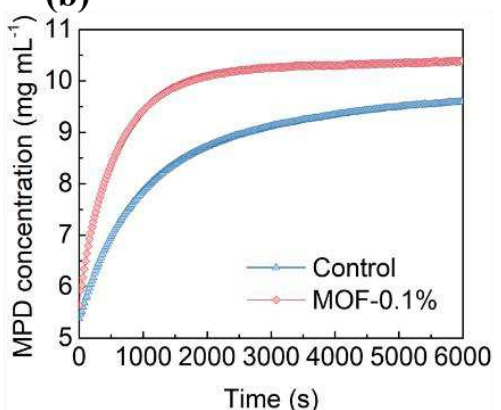

(e)

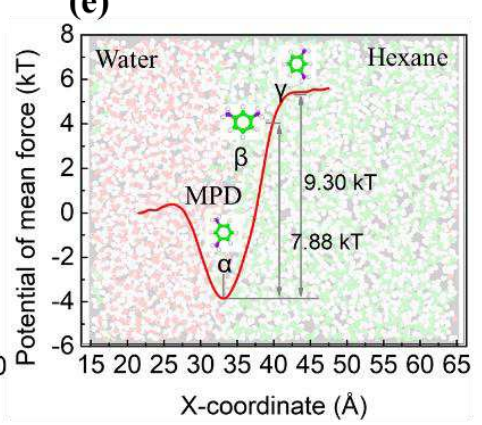

(c) $\downarrow_{\mathrm{A}} \quad \downarrow_{\mathrm{A}}$
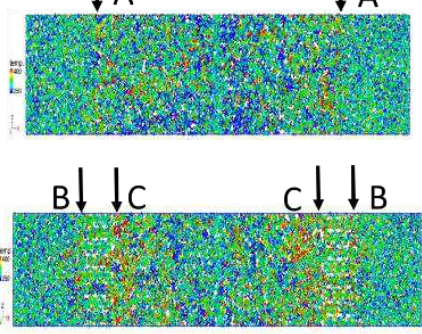

(f)

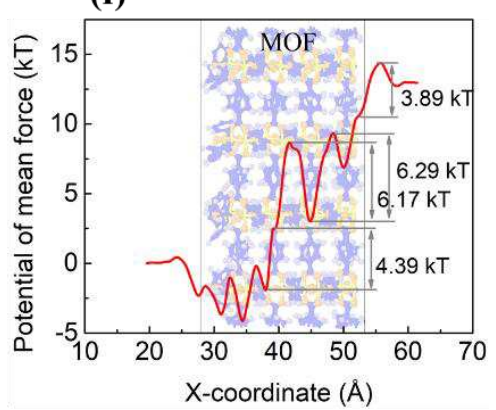

Figure 4. (a) The average heat release rate produced by the addition of $T M C /$ hexane solution into MPD/water solution without (control) and with MOF nanoflakes (MOF-0.1\%) measured by ITC analysis (negative heat release rate represents the exothermal behavior); (b) hexane phase concentration of MPD, which increased due to the trans-interface diffusion from the aqueous phase with/without the addition of MOF nanoflakes; (c) atom temperature distribution after $4 \mathrm{~ns}$ of heat input for water/hexane system (top), where arrow A refers to the water/hexane interface position; and atom temperature distribution after 4 ns of heat input for water/MOF/hexane system (bottom), where arrows $\mathrm{B}$ and $\mathrm{C}$ refer to the water/MOF and $\mathrm{MOF} / \mathrm{hexane}$ interface, respectively; (d) temperature evolution at the interface in water/hexane system and water/MOF/hexane system; (e) MD simulation of the potential of mean force with the MPD molecule at different locations along the X- 
coordinate in water/hexane system; and (f) MD simulation of the potential of mean force with the MPD molecule at different locations along the X-coordinate for water/MOF/hexane system.

The exothermal nature of IP process plays a critical role in PA nanofilm formation ${ }^{50,51}$. The heat release rate is closely related to PA nanofilm formation, and the intensive heat release accelerates the whole reaction process and enhances the cross-linking degree of the polymer matrix ${ }^{52}$. The local temperature rise could lead to "interfacial boiling", facilitating degassing of the solution to generate bubbles that led to the formation of nanovoids structure in the resulting PA film ${ }^{28}$. The heat release rate during IP process, measured by isothermal titration calorimetry (ITC), is shown in Figure 4a and Supplementary Figure 14. With MOF nanoflakes at the interface, the heat release rate was remarkably higher than that of the control sample (Supplementary Figure 14). The addition of MOF nanoflakes nearly tripled the average heat release rate compared to that of the control FIP reaction (Figure 4a). Compared with other porous nanomaterials with high thermal conductivity (e.g., carbon nanotubes ${ }^{53}$, graphene oxide ${ }^{54}$, and metal foam $\left.{ }^{55}\right)$, MOF nanoflakes have a significantly lower thermal conductivity of $0.2231 \mathrm{~W} \mathrm{~m}^{-1} \mathrm{~K}^{-1}$ and thus impose a substantially higher resistance against heat dissipation towards aqueous phase during IP process. The heat retained by MOF nanoflakes accelerated the IP process which in turn released more heat, creating a positive feedback loop to promote a highly vigorous IP process. The accelerated IP process substantially improved the cross-linking degree (and thus the selectivity) of the resulting PA nanofilms. Moreover, with the 2D interfacial MOF self-assembly, the generated heat and nanobubbles were trapped between PA film and MOF nanoflakes during MARIP. The confined heat and nanobubbles drove the MPD 
monomers to the less dense area of the nascent nanofilm where the MPD further reacted with TMC to eliminate defects (i.e., spontaneous self-healing) ${ }^{56}$ and promote the formation of a defect-free PA film that is highly crosslinked throughout.

During the IP process, MPD monomers diffuse from aqueous phase to the organic phase to form the PA nanofilm in organic phase near the interface ${ }^{29}$. The diffusivity of amine monomers in the reaction zone is of critical importance, i.e., a higher diffusivity benefits faster and more complete IP, leading to the formation of a denser PA matrix ${ }^{57}$. The diffusivity of MPD from water to hexane phase was measured by monitoring the MPD concentration in hexane by UV-vis spectrophotometry with/without the presence of MOF nanoflakes. The diffusivity of MPD to hexane phase increased significantly in the presence of MOF nanoflakes (Figure 4b), which was attributed to the reduced energy barrier for MPD transport caused by MOF nanoflakes. The periodic distribution of hydrophilic $\mathrm{Cu}-\mathrm{O}$ groups and hydrophobic benzene ring groups of the MOF nanoflakes changed the energy landscape for MPD diffusion (Figure 4f), thereby facilitating the trans-interfacial transport of MPD monomers and enhancing the kinetics of the IP process ${ }^{29}$. Moreover, the negative charged MOF nanoflakes $(-16.3 \pm 0.8 \mathrm{mV})$ facilitated the accumulation of the positively charged MPD molecules at the water/hexane interface, leading to a greater initial concentration gradient to drive the trans-interfacial transport ${ }^{29}$. The intensified IP due to the rise of local temperature and the enhancement in MPD transport led to the formation of the dense PA active layers.

We further performed molecular dynamics (MD) simulation of heat dissipation across the water/hexane interface in the presence of MOF. After $4 \mathrm{~ns}$ of the heat input, the atoms near the MOF/hexane system had higher temperature with a uniform distribution (Figure 
4c). The equilibrium temperature at the interface in the water/MOF/hexane system was significantly higher than that of the water/hexane system (Figure 4d), which was mainly due to the larger interfacial thermal resistance in the presence MOF (Supplementary Movie 2). For water/hexane system, the transfer of heat produced in IP was mainly resisted by the water/hexane interface with a thermal resistance of $4.38 \times 10^{-9} \mathrm{~m} \mathrm{~K} \mathrm{~W}^{-1}$ (Supplementary Table 2). In the water/MOF/hexane system, however, the heat transfer was resisted by the $\mathrm{MOF} /$ hexane interface, $\mathrm{MOF}$, and MOF/water interface with a total thermal resistance of $1.39 \times 10^{-8} \mathrm{~m} \mathrm{~K} \mathrm{~W}^{-1}$ (Supplementary Table 3 ). The simulation confirmed that the presence of interfacial MOF hinders the heat dissipation, which benefits the formation of denser polyamide nanofilm.

Molecular dynamic simulation was also performed to evaluate the free energy barrier for MPD transport through the water/hexane and water/MOF/hexane interfaces. The simulation shows that MPD molecule has the lowest free energy at the water/hexane interface (Figure 4e), which can be explained by the fact that the hydrophilic amino groups of MPD could form strong hydrogen bonds with water while the hydrophobic benzene rings have a higher affinity to hexene. As a result, the MPD molecules tend to assume a ' a' configuration at the interface that leads to a lower free energy. Once the MPD molecules transport across the interface into the hexane phase, they assume a ' $\beta$ ' configuration with which one amino group enters the hexane phase with another remaining in water. When the MPD molecule completely entered the hexane phase, it changed to ' $\gamma$ ' configuration. Based on the position and configuration (which depends on position), we can define three states, i.e., $\alpha, \beta$, and $\gamma$ states for the MPD molecules. 
The results from molecular dynamic simulation suggest that the $\gamma$ state is unstable, i.e., the spontaneous transport of a single MPD molecule across the interface (without concentration gradient sustained by reaction in the hexane phase) is energetically unfavorable. The MPD molecule is most energetically favorable at the interface with a significant global energy minimum. The transport of an MPD molecule from the interface to the hexane phase involves an energy penalty of $9.30 \mathrm{kT}$ at $25^{\circ} \mathrm{C}$ (Figure $4 \mathrm{e}$ ). The addition of the MOF nanoflakes changes the energy landscape for interfacial MDP diffusion. While the overall energy penalty for MPD transport to the hexane phase from the aqueous phase or from the interface is not reduced, the presence of the MOF nanoflakes at the water/hexane interface creates many local energy minima in the free energy curve that now becomes highly "zigzagging” (Figure 4f). These local energy minima result in metastable states with relatively small energy penalties in-between, thereby facilitating the transinterface transport of MPD.

As the pore size of MOF is similar to the molecular size of MPD (Supplementary Figure $19 \mathrm{a}$ and $19 \mathrm{~b}$ ), the zigzagging of the free energy curve is likely attributable to the periodic distribution of hydrophilic $\mathrm{Cu}-\mathrm{O}$ groups and hydrophobic benzene ring groups of MOFs along the transport direction. While the strong molecular attraction between amino groups of MPD and $\mathrm{Cu}-\mathrm{O}$ groups of MOF nanoflakes reduced the free energy, the repulsion between amino groups and benzene ring groups increased the free energy. In the presence of multiple local minima, the transport of MPD across the water/hexane interface only needs to overcome the largest of the energy penalties between two adjacent minima, which is considerably ( 48\%) lower than that in an MOF-free system. In other words, the reduced 
maximum energy penalty due to the presence of interfacial MOF assembly facilitates transport of MPD across the interface.

Interestingly, the simulation also shows that MPD molecules transport through the MOF framework via a 'flipping' motion (Supplementary Movie 3), which might be attributed to the benzene ring rotation under the thermal disturbance (Supplementary Figure 19c and 19d). Such 'flipping' facilitates MPD molecules to overcome the periodic potential barrier, which benefits the rapid transport of MPD. The simulation results are consistent with experimental observation of increased MPD diffusivity in the presence of MOF nanoflakes (Figure 4b). We note that regardless of the presence of the interfacial MOF assembly, there always exists a positive energy penalty for the trans-interface transport of MPD alone. In a real IP process, however, the polymerization reaction in the hexane phase consumes the MPD and maintains a concentration gradient to sustain trans-interface transport. While molecular dynamic simulations considering all aspects of the IP process are computationally prohibitive, the results supporting MOF-facilitated transport in the absence of polymerization should equally apply in the presence of polymerization.

In summary, we have developed a MOF assembly regulated IP process to fabricate highly crosslinked and ultra-selective PA membranes with performance transcending the upper bound of perm-selectivity tradeoff. Not only these membranes have a substantially higher water permeance compared with that of commercial TFC-PA membranes $(\sim 125 \%$ enhancement), they also achieved unprecedentedly high rejections ( $>90 \%)$ of small neutral molecules (e.g., boron and NDMA) that are regulated but challenging for existing RO membranes to remove. These PA membranes from MARIP can produce water meeting the stringent standard for potable water quality with a single-pass RO, leading to significant 
potential of cost-saving for seawater desalination and wastewater reuse. The mechanisms for forming these high-performance membranes in the presence of amphiphilic MOF nanoflakes include the confinements of trans-interface heat transfer and gas nanobubbles formed in-situ, and the enhancement of the trans-interface transport of diamine monomers. These factors together promote the formation of a highly cross-linked, ultra-thin, and corrugated PA active layer that is both highly water permeable and exceptional in rejecting salts and micropollutants.

\section{METHODS}

Preparation of MOF-regulated free-standing membrane. The free-standing polyamide membrane was prepared by fabricating a polyamide active layer at a free aqueous-organic interface, i.e., via free interface polymerization (FIP). Briefly, for the pristine FIP membrane, the MPD aqueous solution (2.0 wt.\%) was added to a glass container, followed by carefully pouring $0.10 \mathrm{wt} . \% \mathrm{TMC} /$ hexane solution onto the surface of MPD solution. The total reaction time for interfacial polymerization was $1 \mathrm{~min}$. The resultant PA active layer was picked-up on a substrate and rinsed with sufficient hexane to remove the unreacted TMC. The PA active layer was then floated on a water surface and transferred to PES membrane. For the MOF-regulated free-standing membrane (see Supplementary Figure 4), before the adding of TMC/hexane solution, the MOF/hexane solution ( $0.05 \mathrm{wt} . \%$, $0.10 \mathrm{wt} . \%$, and $0.20 \mathrm{wt} . \%$ ) was carefully spread onto the surface of MPD solution, ensuring the surface was fully covered by the MOF/hexane solution. The system was then placed at room temperature for 10 min until all the hexane was evaporated, which enabled the MOF nanoflakes to assemble at the interface ${ }^{58}$. Then $\mathrm{TMC} /$ hexane solution was poured onto the 
surface of aqueous solution to initiate the interfacial polymerization reaction. The obtained membranes using MOF assembly regulated IP (MARIP) are denoted as MARIP-0.05, MARIP-0.1 and MARIP-0.2 membranes with MOF dosages of 0.05 wt. $\%, 0.1$ wt.\% and 0.2 wt. $\%$, respectively.

Conventional TFC membrane preparation. The conventional TFC membrane was fabricated using a conventional IP method according to our previous work ${ }^{59}$. Briefly, the PES substrate was first immersed in $2.0 \mathrm{wt} . \%$ MPD aqueous solution for $2 \mathrm{~min}$. The excess MPD solution was removed by nitrogen gas using an air knife. Then the 0.10 wt.\% TMC/hexane solution was poured onto the substrate surface for $45 \mathrm{~s}$, resulting in the formation of polyamide nanofilm on the substrate. After thoroughly rinsing with hexane, the membrane was cured at $75^{\circ} \mathrm{C}$ for $5 \mathrm{~min}$. The resultant membrane (TFC-PA) was stored in DI water at $4^{\circ} \mathrm{C}$ for further characterization and tests.

MOF-mixed free-standing membrane preparation. The MOF-mixed free-standing membrane was fabricated using the procedures similar to those in the MOF-regulated method as described above, except for the lack of evaporation process. The TMC/hexane solution was immediately (within $5 \mathrm{~s}$ ) added to the system after the pouring of MOF/hexane solution to exclude the assembling process. The concentration of MOF/hexane solution was $0.1 \mathrm{wt} . \%$. The obtained membrane is denoted as Mix-MOF membrane.

Characterization methods. Top surface, bottom surface, and cross-section morphologies of the membranes were observed by SEM (S4800, Hitachi, Japan). The TEM (TALOS F200X, Thermofisher Scientific, U.S.) was used to examine the membrane cross-sectional images. The intrinsic structure of the polyamide nanofilm including apparent thickness $\left(\delta_{\text {app }}\right)$, intrinsic thickness $\left(\delta_{\text {int }}\right)$, surface area ratio (SAR), and void volume fraction $(f)$ were 
determined from TEM images using the ImageJ software. A quartz crystal microbalance (QCM, Q-sense E4, Biolin Scientific, Sweden) was used to quantify the structural changes (i.e., $\delta_{\text {int }}$ and $f$ ) in PA nanofilms ${ }^{59}$. The details of the TEM and QCM analysis can be found in Supplementary Materials.

The microstructure of PA films was characterized by a variable monoenergy slow positron beam coupled DBES (Institute of high energy physics, Beijing, China). The DBES spectrum shows the information about the positron annihilation event, where the depth profile of the cavity size distribution in polyamide films can be characterized by the $S$ parameter $^{60}$. The water/hexane interfacial tensions were analyzed using an automatic surface tension meter (K100C-MK2, Krüss, Germany) following Wilhelmy plate procedure $^{61}$.

To measure heat release rate during the interfacial polymerization reaction, isothermal titration calorimetry (Microcal PEAQ-ITC, Malvern, UK) was used to measure the exothermic reaction at the interface. Briefly, $100 \mu \mathrm{L}$ of $2.0 \mathrm{wt} . \% \mathrm{MPD} /$ water solution was placed in the cell. Then $0.1 \mathrm{wt} . \% \mathrm{TMC} /$ hexane solution $(40 \mu \mathrm{L})$ was poured in the syringe. The heat release rate was monitored in every injection of $\mathrm{TMC} / \mathrm{hexane}$ to $\mathrm{MPD} /$ water solution. For the MOF nanoflakes addition, before the titration, $10 \mu \mathrm{L} 0.10$ wt.\% $\mathrm{MOF} /$ hexane solution was injected into the water surface in the cell. The cell was then kept open under the light until all the hexane was evaporated. Then TMC/hexane solution was injected to measure the heat release rate during interfacial polymerization reaction with the assistance of MOF nanoflakes.

To monitor the diffusion process of MPD monomers from aqueous phase with/without nanoflakes to organic phase through interface, UV-vis spectra-photometer (TU1810, 
PERSEE, China) was modified, with the height of UV-light $1 \mathrm{~mm}$ above the water/hexane interface ${ }^{62}$. The change in absorbance at the monitoring point was recorded every $30 \mathrm{~s}$ once the hexane solution was added into the system. For the MOF addition, 0.10 wt.\% MOF/hexane solution was added to fully cover the surface of aqueous phase and evaporated thoroughly before the addition of hexane solution.

Separation performance tests. Membrane separation performance was tested using a laboratory-scale crossflow filtration setup at 16.0 bar and $24 \pm 0.1^{\circ} \mathrm{C}$ as described in our previous study ${ }^{63}$. A membrane coupon $\left(6.25 \mathrm{~cm}^{2}\right)$ was pre-compacted with DI water at a crossflow rate of $22.4 \mathrm{~cm} \mathrm{~s}^{-1}$ for $6 \mathrm{~h}$ before the measurement of water flux and salt rejection. Water flux (Eq. (1)) was calculated by weighing the mass of the permeate over time.

$$
J_{\mathrm{W}}=\frac{\Delta m}{A_{\mathrm{m}} \times \Delta t \times \rho}
$$

where $J_{\mathrm{W}}\left(\mathrm{L} \cdot \mathrm{m}^{-2} \cdot \mathrm{h}^{-1}\right)$ is water flux, $\Delta m(\mathrm{~g})$ is the mass of the permeate collected over a designated time interval $(\Delta t, \mathrm{~h}), A_{\mathrm{m}}$ is the membrane surface area $\left(\mathrm{m}^{2}\right)$, and $\rho\left(\mathrm{kg} \cdot \mathrm{L}^{-1}\right)$ is the density of water. The membrane water permeance $(A)$ was determined as the ratio of water flux over the applied pressure.

The salt rejection was tested using a feed solution containing $2000 \mathrm{mg} \mathrm{L}^{-1} \mathrm{NaCl}$. The salt rejection $(R)$ was determined according to Eq. (2):

$$
R=\left(1-\frac{C_{\mathrm{p}}}{C_{\mathrm{f}}}\right) \times 100 \%
$$

where the conductivity of the feed $C_{\mathrm{f}}\left(\mathrm{mg} \mathrm{L}^{-1}\right)$ and permeate $C_{\mathrm{p}}\left(\mathrm{mg} \mathrm{L}^{-1}\right)$ were determined using an electrical conductivity meter (Cole-Parmer, USA).

The salt permeability coefficient (B) was determined using Eq. (3): 


$$
B=J_{\mathrm{W}} \frac{1-R}{R} \exp \left(-\frac{J_{\mathrm{W}}}{k}\right)
$$

where the term $\exp \left(-\frac{J_{\mathrm{W}}}{k}\right)$ is used to correct the concentration polarization effect, and $k$ is the mass transfer coefficient in the crossflow cell calculated according to She et al. ${ }^{64}$

To assess the membrane separation performance, we further measured the rejection of the inorganic micropollutant (boron, $\mathrm{Mw}=61.8 \mathrm{~g} \mathrm{~mol}^{-1}$ ) and the very small neutral micropollutant (NDMA, Mw=74.1 $\left.\mathrm{g} \mathrm{mol}^{-1}\right)$. The concentration of boron $\left(5 \mathrm{mg} \mathrm{L}^{-1}\right.$, at $\mathrm{pH}$ 6) was determined by an inductive coupled plasma optical emission spectrometer (ICPOES, Agilent 5110). ${ }^{22}$ The NDMA rejection was analyzed by a liquid chromatograph mass spectrometer $^{65}$ (LC-MS, U3000-QE, Thermo) with the feed solution of $890 \mu \mathrm{g} \mathrm{L}^{-1}$. The elevated feed concentration was proved to have no significant influence on NDMA removal $^{66}$, and could be detected by LC-MS directly without sample concentration. The NDMA-D6 $(\mathrm{m} / \mathrm{z}=81)$ was used as internal standard sample for quantification.

Computation simulation. To explore the effect of the MOF nanoflakes on the dissipation of reaction heat from the MOF/hexane interface to the water and hexane phases, we simulated the heat diffusion process in both water/MOF/hexane and water/hexane systems for comparison. A symmetrical setup of the thermal diffusion system was used in the simulation. Heat was continuously added to the two slabs at a specific rate by changing the velocity of the atoms, with two cold slabs at constant temperatures located in the water and hexane phases, respectively (Supplementary Figure 16). Both the water/hexane system and water/MOF/hexane system contain 4,172 water molecules, 936 hexane molecules, and 100 MPD molecules and 36 TMC molecules in the water and hexane phases, respectively. For water/MOF/hexane system, the MOF nanoflake has a size of $4 \times 6 \times 6$ unit cells with 
periodic conditions in the $y$ - and $z$-directions, which corresponds to a size of about $21 \times 39 \times 43 \AA^{3}$. The slabs for adding heat were in the hexane phase close to the MOF/hexane interface. The releasing rate of heat from the interfacial polymerization between MPD and TMC with an interfacial area of $1,677 \AA^{2}$ was estimated to be $7.0 \times 10^{-7} \mathrm{kcal} \mathrm{mol}^{-1} \mathrm{ps}^{-1}$ according to the bond energy between MPD and TMC. The release rate of heat was determined by quantum calculations in combination with the measured diffusion rate of MPD across the MOF/hexane interface. Considering the sizes of the simulated system and MOF were several orders smaller than that in the experiments, the rate of adding heat to the slabs, $Q_{1}$, was set to $0.70 \mathrm{kcal} \mathrm{mol}^{-1} \mathrm{ps}^{-1}$ to simulate the heat from the exothermic interfacial polymerization reaction. The cold slabs in the water and hexane phases were maintained at a temperature of $298 \mathrm{~K}$ with the Langevin thermostat, and heat would be removed from these two slabs since a microcanonical (NVE) ensemble was applied to the whole system. The damping factor of the Langevin thermostat was set to $0.1 \mathrm{ps}$ according to the work of Li et al. ${ }^{67}$ The amounts of heat removed from these two slabs in every unit time were accumulatively collected. The molecular dynamics simulation was conducted with the Lammps package. All the slabs have a thickness of $5 \AA$. The simulations were run for 4,000,000 steps with a timestep of $1 \mathrm{fs}$. The heat was added to the system every 10 steps, and the removed heat from the cold slabs were collected every 100 steps. Before the simulation of heat diffusion, the system had been fully equilibrated in an NPT ensemble at a temperature of $298 \mathrm{~K}$ and a pressure of $1 \mathrm{~atm}$. Since the system was symmetric in the $\mathrm{x}$ direction, we averaged the temperature profile of the system in the $\mathrm{x}$-direction.

To get the insight of trans-interface resistance of MPD from water to hexane with and without MOF, the combination of Umbrella Sampling ${ }^{68}$ and weighted histogram analysis 
method (WHAM) ${ }^{69,70}$ was used to calculate the necessary free energy of MPD that transported through water/hexane interface. The systems were simulated with NVE thermodynamic ensemble at $298 \mathrm{~K}$ temperature. Two simulated systems were constructed, i.e., one is water/hexane system, and the other is water/MOF/hexane system. Both simulated lattice cell is comprised of the same numbers of $\mathrm{H}_{2} \mathrm{O}(1,043)$ and MPD (1) on the left, and hexane (234) on the right. In the MD model with MOF, the MOF nanoflake that is placed between water and hexane has the size of $4 \times 6 \times 6$ units cell and $21 \AA \times 39 \AA \times 43$ $\AA$, with periodicity in the $y$ - and $z$-coordinate directions. Both lattice cell was separated into several sampling segments with the length of $0.5 \AA$ (near the interface) and $1 \AA$ (far from interface) (Supplementary Figure 18). Each sampling segment consists of 1,000,000 steps, and each step lasts 1 femtosecond. During the MD process, the MPD molecule was initially fixed at the center of each sampling segment with a spring potential, and then the simulations were operated to obtain the final equilibrium state for umbrella sampling. The elastic coefficient of the spring used in Umbrella Sampling was set to 3-10 $\mathrm{kcal} \mathrm{mol}^{-1} \AA^{-2}$.

\section{REFERENCES}

1. Park, H.B. et al. Maximizing the Right Stuff: The Trade-Off between Membrane Permeability and Selectivity. Science 356, eaab0530 (2017).

2. Mueller, J.T. \& Gasteyer, S. The Widespread and Unjust Drinking Water and Clean Water Crisis in the United States. Nat. Commun. 12, 3544 (2021).

3. Elimelech, M. \& Phillip, W.A. The Future of Seawater Desalination: Energy, Technology, and the Environment. Science 333, 712 (2011).

4. Liang, C.Z. et al. Ultra-Strong Polymeric Hollow Fiber Membranes for Saline Dewatering and Desalination. Nat. Commun. 12, 2338 (2021). 
5. Fujioka, T. et al. High Rejection Reverse Osmosis Membrane for Removal of N-Nitrosamines and Their Precursors. Water Res. 131, 45-51 (2018).

6. Lim, Y.J. et al. Seawater Desalination by Reverse Osmosis: Current Development and Future Challenges in Membrane Fabrication - a Review. J. Membr. Sci. 629, 119292 (2021).

7. Tang, C.Y. et al. Potable Water Reuse through Advanced Membrane Technology. Environ. Sci. Technol. 52, 10215-10223 (2018).

8. DHS. California Department of Health Services; Ndma in California Drinking Waterr; March 15. 181, (2002).

9. Fujioka, T. et al. Plugging Nonporous Polyamide Membranes for Enhanced Rejection of Small Contaminants During Advanced Wastewater Treatment. Sep. Purif. Technol. 253, 117490 (2020).

10. Fujioka, T. et al. Rejection of Small and Uncharged Chemicals of Emerging Concern by Reverse Osmosis Membranes: The Role of Free Volume Space within the Active Skin Layer. Sep. Purif. Technol. 116, 426-432 (2013).

11. Fujioka, T. et al. Transport of Small and Neutral Solutes through Reverse Osmosis Membranes: Role of Skin Layer Conformation of the Polyamide Film. J. Membr. Sci. 554, 301-308 (2018).

12. Fujioka, T. et al. N-Nitrosamine Removal by Reverse Osmosis for Indirect Potable Water Reuse - a Critical Review Based on Observations from Laboratory-, Pilot- and Full-Scale Studies. Sep. Purif. Technol. 98, 503-515 (2012).

13. Werber, J.R. et al. The Critical Need for Increased Selectivity, Not Increased Water Permeability, for Desalination Membranes. Environ. Sci. Technol. Lett. 3, 112-120 (2016).

14. Marron, E.L. et al. A Tale of Two Treatments: The Multiple Barrier Approach to Removing Chemical Contaminants During Potable Water Reuse. Acc. Chem. Res. 52, 615-622 (2019).

15. Fujioka, T. et al. Transport of N-Nitrosamines through a Reverse Osmosis Membrane: Role of Molecular Size and Nitrogen Atoms. Environ. Sci. Technol. Lett. 6, 44-48 (2018). 
16. Redondo, J. et al. Boron Removal from Seawater Using Filmtectm High Rejection Swro Membranes. Desalination 156, 229-238 (2003).

17. Tu, K.L. et al. Boron Removal by Reverse Osmosis Membranes in Seawater Desalination Applications. Sep. Purif. Technol. 75, 87-101 (2010).

18. Fujioka, T. et al. Impact of Heat Modification Conditions on the Removal of NNitrosodimethylamine by Polyamide Reverse Osmosis Membranes. Sep. Purif. Technol. 247, $116921(2020)$.

19. Khoramian, R. et al. Graphene Oxide Nanosheets for Oil Recovery. ACS Appl. Nano Mater. 2, 5730-5742 (2019).

20. Ali, Z. et al. Defect-Free Highly Selective Polyamide Thin-Film Composite Membranes for Desalination and Boron Removal. J. Membr. Sci. 578, 85-94 (2019).

21. Wang, H. et al. Membrane Adsorbers with Ultrahigh Metal-Organic Framework Loading for High Flux Separations. Nat. Commun. 10, 4204 (2019).

22. Yang, Z. et al. Hydrophilic Silver Nanoparticles Induce Selective Nanochannels in Thin Film Nanocomposite Polyamide Membranes. Environ. Sci. Technol. 53, 5301-5308 (2019).

23. Xie, Q. et al. A Novel Double-Modified Strategy to Enhance the Performance of Thin-Film Nanocomposite Nanofiltration Membranes: Incorporating Functionalized Graphenes into Supporting and Selective Layers. Chem. Eng. J. 368, 186-201 (2019).

24. Rodenas, T. et al. Metal-Organic Framework Nanosheets in Polymer Composite Materials for Gas Separation. Nat. Mater. 14, 48-55 (2015).

25. Werber, J.R. et al. A Path to Ultraselectivity: Support Layer Properties to Maximize Performance of Biomimetic Desalination Membranes. Environ. Sci. Technol. 52, 10737-10747 (2018).

26. Werber, J.R. \& Elimelech, M. Permselectivity Limits of Biomimetic Desalination Membranes. Sci. Adv. 4, eaar8266 (2018). 
27. Ritt, C.L. et al. Ionization Behavior of Nanoporous Polyamide Membranes. Proc. Natl. Acad. Sci. U. S. A. 117, 30191-30200 (2020).

28. Ma, X.-H. et al. Nanofoaming of Polyamide Desalination Membranes to Tune Permeability and Selectivity. Environ. Sci. Technol. Lett. 5, 123-130 (2018).

29. Liang, Y. et al. Polyamide Nanofiltration Membrane with Highly Uniform Sub-Nanometre Pores for Sub-1 a Precision Separation. Nat. Commun. 11, 2015 (2020).

30. de Leon, A.C. et al. Distinct Chemical and Physical Properties of Janus Nanosheets. ACS Nano 11, 7485-7493 (2017).

31. Wang, J. et al. Electrochemical Energy Storage Performance of 2d Nanoarchitectured Hybrid Materials. Nat. Commun. 12, 3563 (2021).

32. Khoee, S. \& Karimi, M.R. Dual-Drug Loaded Janus Graphene Oxide-Based Thermoresponsive Nanoparticles for Targeted Therapy. Polymer 142, 80-98 (2018).

33. Yang, J. et al. Stimuli-Responsive Janus Mesoporous Nanosheets Towards Robust Interfacial Emulsification and Catalysis. Mater. Horiz. 7, 3242-3249 (2020).

34. Luo, D. et al. Nanofluid of Graphene-Based Amphiphilic Janus Nanosheets for Tertiary or Enhanced Oil Recovery: High Performance at Low Concentration. Proc. Natl. Acad. Sci. U. S. A. 113, 7711-6 (2016).

35. Qu, M. et al. Preparation and Interfacial Properties of Ultralow Concentrations of Amphiphilic Molybdenum Disulfide Nanosheets. Ind. Eng. Chem. Res. 59, 9066-9075 (2020).

36. Song, P. et al. 2d and 3d Metal-Organic Framework at the Oil/Water Interface: A Case Study of Copper Benzenedicarboxylate. Adv. Mater. Interfaces 6, 1801139 (2019).

37. Dorvee, J.R. et al. Manipulation of Liquid Droplets Using Amphiphilic, Magnetic OneDimensional Photonic Crystal Chaperones. Nat. Mater. 3, 896-899 (2004).

38. Vanhook, S.J. et al. Long-Wavelength Surface-Tension-Driven Bénard Convection: Experiment and Theory. J. Fluid Mech. 345, 45-78 (1997). 
39. Song, X. et al. Intrinsic Nanoscale Structure of Thin Film Composite Polyamide Membranes: Connectivity, Defects, and Structure-Property Correlation. Environ. Sci. Technol. 54, 3559-3569 (2020).

40. Yang, Z. et al. Mechanistic Insights into the Role of Polydopamine Interlayer toward Improved Separation Performance of Polyamide Nanofiltration Membranes. Environ. Sci. Technol. 54, $11611-11621(2020)$.

41. Pacheco, F. et al. 3d Visualization of the Internal Nanostructure of Polyamide Thin Films in Ro Membranes. J. Membr. Sci. 501, 33-44 (2016).

42. Karan, S. et al. Sub-10 Nm Polyamide Nanofilms with Ultrafast Solvent Transport for Molecular Separation. Science 348, 1347 (2015).

43. Song, X. et al. Intrinsic Nanoscale Structure of Thin Film Composite Polyamide Membranes: Connectivity, Defects, and Structure-Property Correlation. Environ. Sci. Technol. 54, 35593569 (2020).

44. Jeon, I. et al. Janus Graphene: Scalable Self-Assembly and Solution-Phase Orthogonal Functionalization. Adv. Mater. 31, 1900438 (2019).

45. Gong, G. et al. New Insights into the Role of an Interlayer for the Fabrication of Highly Selective and Permeable Thin-Film Composite Nanofiltration Membrane. ACS Appl. Mater. Interfaces 11, 7349-7356 (2019).

46. Tung, K.-L. et al. Characterization of Multilayer Nanofiltration Membranes Using Positron Annihilation Spectroscopy. J. Membr. Sci. 343, 147-156 (2009).

47. Prats, D. et al. Analysis of the Influence of $\mathrm{Ph}$ and Pressure on the Elimination of Boron in Reverse Osmosis. Desalination 128, 269-273 (2000).

48. Liu, L. et al. Thin Film Nanocomposite Reverse Osmosis Membrane Incorporated with Uio-66 Nanoparticles for Enhanced Boron Removal. J. Membr. Sci. 580, 101-109 (2019).

49. Tan, Z. et al. Polyamide Membranes with Nanoscale Turing Structures for Water Purification. Science 360, 518 (2018). 
50. Freger, V. Kinetics of Film Formation by Interfacial Polycondensation. Langmuir 21, 18841894 (2005).

51. Freger, V. Nanoscale Heterogeneity of Polyamide Membranes Formed by Interfacial Polymerization. Langmuir 19, 4791-4797 (2003).

52. Ukrainsky, B. \& Ramon, G.Z. Temperature Measurement of the Reaction Zone During Polyamide Film Formation by Interfacial Polymerization. J. Membr. Sci. 566, 329-335 (2018).

53. Zhang, N. et al. Latent Heat Thermal Energy Storage Systems with Solid-Liquid Phase Change Materials: A Review. Adv. Eng. Mater. 20, 1700753 (2018).

54. Wang, J. et al. Construction of Cnt@Cr-Mil-101-Nh2 Hybrid Composite for Shape-Stabilized Phase Change Materials with Enhanced Thermal Conductivity. Chem. Eng. J. 350, 164-172 (2018).

55. Cui, H.T. Experimental Investigation on the Heat Charging Process by Paraffin Filled with High Porosity Copper Foam. Appl. Therm. Eng. 39, 26-28 (2012).

56. Song, X. et al. Confined Nanobubbles Shape the Surface Roughness Structures of Thin Film Composite Polyamide Desalination Membranes. J. Membr. Sci. 582, 342-349 (2019).

57. Li, Y. et al. Preparation of Thin Film Composite Nanofiltration Membrane with Improved Structural Stability through the Mediation of Polydopamine. J. Membr. Sci. 476, 10-19 (2015).

58. Zhao, S. et al. Janus Mxene Nanosheets for Macroscopic Assemblies. Mat. Chem. Front. 4, 910-917 (2020).

59. Wen, Y. et al. Metal-Organic Framework Nanosheets for Thin-Film Composite Membranes with Enhanced Permeability and Selectivity. ACS Appl. Nano Mater. 3, 9238-9248 (2020).

60. Gan, B. et al. Ultrathin Polyamide Nanofilm with an Asymmetrical Structure: A Novel Strategy to Boost the Permeance of Reverse Osmosis Membranes. J. Membr. Sci. 612, 118402 (2020).

61. Rapp, B.E. Microfluidics: Modelling, Mechanics and Mathematics. Elsevier (2017).

62. Wang, Z. et al. Controlling Structure and Properties of Polyamide Nanofilms by Varying Amines Diffusivity in Organic Phase. J. Membr. Sci. 574, 1-9 (2019). 
63. Wen, Y. et al. Thin-Film Nanocomposite Membranes Incorporated with Water Stable MetalOrganic Framework Cubttri for Mitigating Biofouling. J. Membr. Sci. 582, 289-297 (2019).

64. She, Q. et al. Effect of Feed Spacer Induced Membrane Deformation on the Performance of Pressure Retarded Osmosis (Pro): Implications for Pro Process Operation. Journal of Membrane Science 445, 170-182 (2013).

65. Croll, H. et al. Graphene Oxide Surface Modification of Polyamide Reverse Osmosis Membranes for Improved N-Nitrosodimethylamine (Ndma) Removal. Sep. Purif. Technol. 210, 973-980 (2019).

66. Miyashita, Y. et al. Removal of N -Nitrosamines and Their Precursors by Nanofiltration and Reverse Osmosis Membranes. J. Environ. Eng. 135, 788-795 (2009).

67. Li, Z. et al. Influence of Thermostatting on Nonequilibrium Molecular Dynamics Simulations of Heat Conduction in Solids. The Journal of Chemical Physics 151, 234105 (2019).

68. Torrie, G.M. \& Valleau, J.P. Nonphysical Sampling Distributions in Monte Carlo Free-Energy Estimation: Umbrella Sampling. Journal of Computational Physics 23, 187-199 (1977).

69. Souaille, M. \& Roux, B. Extension to the Weighted Histogram Analysis Method: Combining Umbrella Sampling with Free Energy Calculations. Comput. Phys. Commun. 135, 40-57 (2001).

70. Kästner, J. \& Thiel, W. Analysis of the Statistical Error in Umbrella Sampling Simulations by Umbrella Integration. The Journal of Chemical Physics 124, 234106 (2006).

\section{Acknowledgements}

This work was supported by the National Natural Science Foundation of China (51838009 and 51925806). 


\section{Author contributions}

Y.W. and Z.W. conceived the idea and designed the research. Y.W. performed the experiment including membrane fabrication, characterization and performance test. X.C. performed the DBES measurement. Y.W., Z.W., and Z.C.W. carried out the simulation. R.D., X.L, S.L., C.T., Z.C.W. and X.Z provided constructive suggestions for results and discussion. C.T. and S.L. revised the paper. Y.W., S.L., C. T., Z.C.W. and Z.W. contribute to writing the manuscript. All coauthors discussed the results.

\section{Competing interests}

The authors declare no competing interests.

\section{Additional Information}

Supplementary information is available for this paper at...

Correspondence and requests for materials should be addressed to S.L. C.T. or Z.W. 


\section{Supplementary Files}

This is a list of supplementary files associated with this preprint. Click to download.

- Slvffinal0810.pdf

- supplementarymovie1.mp4

- Supplementarymovie2.1withoutCuBDC.mp4

- Supplementarymovie2.2withCuBDC.mp4

- Supplementarymovie3.mp4 